\title{
PAPER
}

\section{Raised plasma oxidised LDL in acute cerebral infarction}

\section{Uno, K T Kitazato, K Nishi, H Itabe, S Nagahiro}

J Neurol Neurosurg Psychiatry 2003;74:312-316

See end of article for authors' affiliations

.....................

Correspondence to: Dr Masaaki Uno, Department of

Neurosurgery, School of

Medicine, The University of

Tokushima, 3-8-15

Kuramoto-cho, Tokushima

770-8503, Japan;

muno@

clin.med.tokushima-u.ac.jp

Received 12 July 2002

In revised form

31 October 2002

Accepted

11 November 2002

.......................
Background: The association between oxidised low density lipoprotein (OxLDL) and cerebral infarction is suspected but not established.

Objectives: To determine whether plasma OxLDL is a useful marker for monitoring oxidative stress in stroke patients.

Methods: Plasma OxLDL concentrations were determined in 56 stroke patients with cerebral infarction $(n=45)$ or cerebral haemorrhage $(n=11)$, and in 19 age matched controls, using a novel sandwich enzyme linked immunosorbent assay.

Results: Compared with the controls $(0.130(0.007) \mathrm{ng} / \mathrm{\mu g}$ LDL, mean (SEM)), OxLDL was significantly raised in patients with cerebral infarction $(0.245(0.022) ; p<0.0001)$ but not in those with haemorrhage $(0.179(0.023))$. Patients with cortical ischaemic infarcts $(n=22)$ had higher OxLDL levels than either the controls $(p<0.0001)$ or the patients with non-cortical ischaemic infarcts $(n=23)$ $(p<0.001)$. Increased OxLDL concentrations in patients with cortical infarcts persisted until the third day after stroke onset. The National Institutes of Health stroke scales in patients with cortical infarction were higher than in those with non-cortical infarction $(p<0.01)$.

Conclusions: There is a significant association between raised plasma OxLDL and acute cerebral infarction, especially cortical infarction. Plasma OxLDL may reflect oxidative stress in stroke patients.
S troke is a leading cause of mortality and of long term disability among adults in industrialised countries. ${ }^{12}$ Early detection and control of risk factors such as hypertension, smoking, and diabetes mellitus are thought to be crucial in reducing the risk of stroke and providing effective care. However, the risk factors may differ depending on the aetiology of the stroke, and some stroke victims have none of the risk factors.

Oxidative stress is characteristic of thrombotic strokes. The event results in the generation of free radicals and oxidative injury, leading to the promotion of lipid peroxidation. This not only affects low density lipoproteins (LDL) and other lipoproteins, but also cellular lipids, including those in the arterial wall and in macrophages. ${ }^{3-5}$ An increase in oxidative products can contribute to atherosclerosis and to altered control of vasomotor tone. The lack of direct evidence on the role of oxidatively modified low density lipoprotein (OxLDL) in patients with ischaemic stroke partly reflects a lack of sensitive and specific tools with which to measure OxLDL levels. Although it is known that OxLDL is present in atherosclerotic lesions and that it has proatherogenic properties, ${ }^{36-10}$ the broad structural spectrum of lipoproteins comprising OxLDL has hampered investigative progress.

Itabe et al have established a murine monoclonal antibody, FOHIa/DLH3 (DLH3), which recognises oxidatively modified lipoproteins in plasma and foam cells in human atherosclerotic lesions. ${ }^{11}{ }^{12}$ This antibody reacts with several oxidised products of phosphatidylcholine, including its aldehyde derivatives, which covalently modify polypeptides. Using it, Itabe et al observed increased plasma OxLDL concentrations in patients with cardiovascular disease. ${ }^{13-15}$ Holvoet et al also found increased plasma OxLDL in patients with atherosclerotic cardiovascular disease clinically manifesting as stable and acute coronary syndrome, and in heart transplant patients. ${ }^{16}{ }^{17}$ They suggested that oxidised LDL might be a marker of cardiovascular disease. The association between coronary and cerebrovascular atherosclerosis can be ascribed to a common pathophysiological antecedent: the lesions tend to develop first in the aorta, then in the coronary arteries, and later in the cerebrovascular and peripheral circulation. ${ }^{18}$
We have observed that coronary artery disease often accompanies carotid artery disease; this association is found in Japanese as well as in Western populations. ${ }^{19}$ We therefore speculated that the plasma OxLDL level in patients with cerebral ischaemic stroke would be increased and could serve as a marker of ischaemic stroke.

In this study, we determined plasma OxLDL concentrations in patients with acute ischaemic stroke using a sandwich enzyme linked immunosorbent assay (ELISA) employing DLH3 and anti-apo B antibodies. At the time of stroke onset and at subsequent intervals over a 30 day period or until discharge, we investigated the relation between the OxLDL concentrations and ischaemic stroke, and evaluated plasma OxLDL as a marker in stroke patients.

\section{METHODS}

\section{Subjects}

The study population consisted of 56 patients (mean (SEM) age 66.9 (1.5) years, range 35 to 84 ) who had experienced a cerebrovascular event. There were 32 men and 24 women. The controls were 19 age matched healthy volunteers who had no history of a cerebrovascular accident (nine men and 10 women, mean age 61.2 (2.4) years, range 34 to 74 ). The patients were consecutive admissions to the department of neurological surgery at the University of Tokushima Hospital between February 2000 and April 2001. Informed consent was obtained from all participants or their relatives.

All patients underwent brain computed tomography (CT) and magnetic resonance imaging (MRI) on admission. Echocardiography and extracranial duplex ultrasound were undertaken at the discretion of the attending clinician. Stroke was diagnosed on the basis of the results of

Abbreviations: $\mathrm{Gl}$, infarcts located in the cerebral cortex (frontal, parietal, and temporal lobes); GIl, infarcts involving other regions of the brain; HDL, high density lipoprotein; LDL, low density lipoprotein; NIHSS, National Institutes of Health stroke scale; OxLDL, oxidised low density lipoprotein; TOAST, trial of Org 10172 in acute stroke treatment 
Table 1 Demographic characteristics of the patients

\begin{tabular}{llll}
\hline Characteristic & Cerebral infarction & Cerebral haemorrhage & Control \\
\hline $\mathrm{n}$ & 45 & 11 & 19 \\
Agge (years) & 35 to 84 & 45 to 78 & 34 to 74 \\
$\quad$ Range & $67.4(1.5)$ & $64.4(3.3)$ & $61.2(2.4)$ \\
Mean (SEM) & $24 / 21$ & $7 / 4$ & $9 / 10$ \\
Male/female & $22(49 \%)$ & 7 & - \\
Hypertension & $22(49 \%)$ & 4 & - \\
Diabetes mellitus & $10(22 \%)$ & 1 & - \\
Atrial fibrillation & $12(27 \%)$ & 2 & - \\
Hyperlipidaemia & $7.7(1.1)$ & $7.6(1.4)$ & $0.130(0.007)$ \\
NIH stroke scale & $0.245(0.022)^{*}$ & $0.179(0.023)$ & $5.51(0.12)$ \\
OxLDL (ng/ul LDL) & $5.07(0.18)$ & $5.06(0.58)$ & $1.43(0.38)$ \\
Total cholesterol (mmol/I) & $1.24(0.05)$ & $1.31(0.06)$ & $1.36(0.16)$ \\
HDL cholesterol (mmol/I) & $3.40(0.17)$ & $3.48(0.31)$ & $(0.26)$ \\
LDL cholesterol (mmol/I) & $1.17(0.08)$ & $1.48(0.22)$ & \\
Triglyceride (mmol/I) & &
\end{tabular}

the neurosurgeons' clinical examinations. A National Institutes of Health stroke scale value (NIHSS) ${ }^{20}{ }^{21}$ was assigned on admission and at 30 days after stroke onset or on discharge. Baseline demographic data (age, sex), a history of conventional vascular risk factors (hypertension, diabetes mellitus, hyperlipidaemia, tobacco use), and a history of previous vascular events (myocardial infarction, atrial fibrillation, angina, intermittent claudication) were obtained. Patients whose pertinent data could not be evaluated at the time of stroke onset were excluded from the study.

Neurosurgeons, blind to the results of the OxLDL level studies, classified the 56 patients into a cerebral infarction group $(n=45)$ and a cerebral haemorrhage group $(n=11)$. Determinations were based on the results of clinical evaluations and imaging studies (table 1). More than $45 \%$ of the patients were hypertensive, regardless of the stroke subtype. Half the patients with cerebral infarcts had diabetes mellitus. Patients with cerebral infarction had a higher rate of atrial fibrillation and hyperlipidaemia than patients with cerebral haemorrhage.

The 45 patients with cerebral infarction were further subdivided into three groups on the basis of their clinical category: cardioembolic infarction $(n=16)$, atherothrombotic infarction $(\mathrm{n}=17)$, and lacunar infarction $(\mathrm{n}=12)$. The stroke subtypes were defined according to the TOAST classification system ${ }^{22}$ : the atherothrombotic infarction group includes patients with clinical and brain imaging findings of either significant stenosis or occlusion of a major brain artery or a branch cortical artery, presumably caused by atherosclerosis; the cardioembolic infarction group includes the patients with arterial occlusion presumably caused by an embolus arising in the heart; the lacunar infarction group includes patients with one of the traditional clinical lacunar syndromes and no evidence of cerebral cortical dysfunction, and also patients whose CT/MRI did not show lesions with a diameter exceeding $1.5 \mathrm{~cm}$.

Using the results of CT or MRI studies, patients with cerebral infarction were further subdivided into two groups according to the site of the infarct: those in whom the infarct was located in cortical regions in the cerebral hemisphere involving the frontal, parietal, and temporal lobe $(\mathrm{GI}, \mathrm{n}=22)$, and those whose infarcts resulted in basal ganglia lesions in the anterior circulation (putamen, corona radiata) or in the posterior circulation (occipital lobe, cerebellum, brain stem, and thalamus) (GII, $\mathrm{n}=23$ ). ${ }^{23}$ MRI or cerebral angiography showed that 19 of the 22 GI patients (86\%) had occlusion or stenosis of the internal carotid artery or the horizontal portion of the middle cerebral artery. Of the 23 GII patients, three $(13 \%)$ had internal carotid artery or middle cerebral artery occlusion or stenosis, and six (26\%) had unilateral vertebral artery or posterior cerebral artery occlusion.

\section{Blood sampling}

Venous blood samples for the OxLDL assay and other biochemical analyses were obtained on admission (within 24 hours after the stroke onset) and again on the mornings of days 3, 7, 14, and 30 after stroke onset, after an overnight fast of at least 12 hours. To measure plasma OxLDL concentrations, blood was drawn into tubes containing EDTA-2Na. These were chilled on ice, centrifuged at $4^{\circ} \mathrm{C}$ for separation, and stored for a maximum of six days at $4^{\circ} \mathrm{C}$ until LDL isolation. Other routine chemical laboratory assays were performed according to protocols established by our clinical laboratory department.

\section{Isolation of LDL}

LDL isolation was done by potassium bromide stepwise density gradient ultracentrifugation, as described previously. ${ }^{11}$ Briefly, plasma was transferred to a 4 PC tube (Hitachi Inc, Tokyo, Japan) and overlaid with $0.14 \mathrm{M} \mathrm{NaCl} / 0.01 \mathrm{M}$ phosphate buffered saline (PBS, pH 7.4), using one third of the plasma sample. The tubes were centrifuged at $350000 \mathrm{~g}$ for 10 minutes at $4^{\circ} \mathrm{C}$ in a CS 120 ultracentrifuge, using an AT 80 rotor (Hitachi). The creamy layer at the top was discarded and the remaining sample was overlain with PBS and centrifuged again at $350000 \mathrm{~g}$ for 3.5 hours at $4^{\circ} \mathrm{C}$. After discarding the top layer, $0.5 \mathrm{~g} / \mathrm{ml} \mathrm{KBr}$ was added to the bottom layer, using one fifth of the sample volume $(\mathrm{d}=1.063)$. This was followed by centrifuging at $350000 \mathrm{~g}$ for 3.5 hours at $4^{\circ} \mathrm{C}$, and the fraction with a density 1.019-1.063 was collected by pipetting. The isolated LDL fraction was dialysed at $4^{\circ} \mathrm{C}$ overnight against PBS ( $\mathrm{pH} 7.4$ ) containing $0.1 \%$ EDTA to remove any remaining $\mathrm{KBr}$. The protein content of the LDL fraction was determined using the BCA protein assay kit (Pierce, Rockford, Illinois, USA). Standard OxLDL was prepared by incubation of LDL with $5 \mu \mathrm{M} \mathrm{CuSO}_{4}$ at $37^{\circ} \mathrm{C}$ for three hours, and anti-OxLDL monoclonal antibody was prepared as described previously. ${ }^{11}$

\section{Determination of plasma OxLDL concentration}

The plasma OxLDL concentration was determined by a competition ELISA assay, a modification of a sandwich ELISA procedure for OxLDL determination. ${ }^{13}$ Briefly, microtitre wells precoated with DLH3 monoclonal antibody $(5 \mu \mathrm{g} / \mathrm{ml}$ in PBS, 100 $\mu \mathrm{l} /$ well) were blocked with $1 \%$ bovine serum albumin (BSA) in $50 \mathrm{mM}$ Tris buffered saline, $\mathrm{pH}$ 8.0. To the wells were added 100 $\mu \mathrm{l}$ of appropriately diluted samples and standards of OxLDL. The microtitre plates were then left at $4^{\circ} \mathrm{C}$ overnight. After washing with Tris buffered saline containing 0.05\% Tween 20, the remaining OxLDL was detected with $100 \mu \mathrm{l}$ of sheep anti-human apoB IgG antibody (Boehringer, Mannheim, Germany) and $100 \mu \mathrm{l}$ of alkaline phosphatase conjugated donkey anti-sheep IgG antibody (Chemicon, Temecula, California, USA). The reactivity of alkaline phosphatase was measured by 


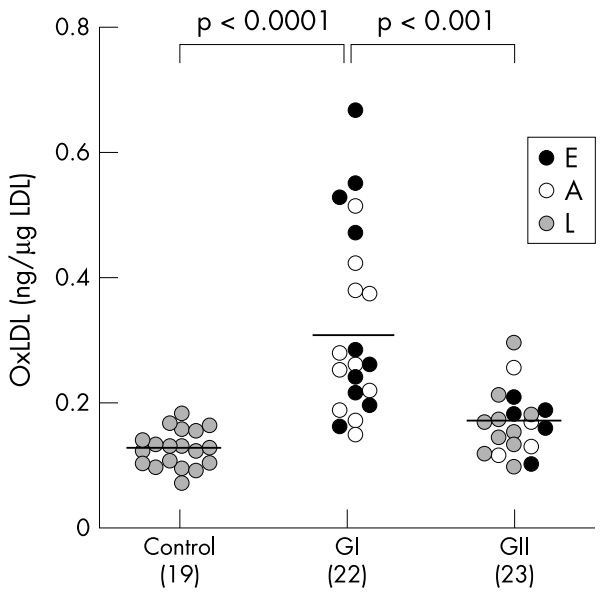

Figure 1 Effect of the stroke type $(E$, cardioembolic; $A$, atherothrombotic; L, lacunar infarction) and the location of the infarct $(\mathrm{Gl}$, cortical lesion in cerebral hemisphere; GII, infarction in the other regions) on the plasma oxidised LDL (OxLDL) concentration. Horizontal bars indicate average value. $p$ Values determined by analysis of variance followed by Scheffé's test.

incubating washed plates for an appropriate length of time at $37^{\circ} \mathrm{C}$ with $100 \mu \mathrm{l}$ substrate solution containing $1 \mathrm{mg} / \mathrm{ml}$ of disodium p-nitrophenyl-phosphate hexahydrate (Wako, Osaka, Japan). Absorbance at $405 \mathrm{~nm}$ was measured using a microplate reader for comparison with a standard curve obtained under the same assay conditions. Simultaneously, a parallel ELISA was run to determine amounts of apo B in the same lipoprotein fractions using anti-apoB monoclonal antibody (OEM, Toms River, New Jersey, USA). The OxLDL levels were expressed as amount of OxLDL per $\mu \mathrm{g}$ of apoB protein (LDL) and recorded at stroke onset and sequentially at appropriate intervals during the follow up period.

\section{Statistics}

Sequentially obtained data, expressed as the mean (SEM), were analysed using the Mann-Whitney U test for a two group comparison; analysis of variance followed by Scheffé's test was used for comparisons of three groups or more. The correlations between OxLDL levels and other clinical chemistry assays or NIHSS score were examined by the Spearman rank correlation test. GI to GII ratios were compared using the $\chi^{2}$ test. Statistical analyses were done on a Macintosh computer running statistical software (Stat View 4.0). Statistical significance was assumed at a probability $(\mathrm{p})$ value of $<0.05$.

\section{RESULTS}

\section{Plasma OxLDL in acute cerebral infarction}

At stroke onset, patients with cerebral infarction had a 1.9 times higher concentration of OxLDL than the controls: 0.245 (0.022) $v 0.130(0.007) \mathrm{ng} / \mathrm{\mu g}$ LDL $(\mathrm{p}<0.01)$ (table 1$)$. There was no difference in OxLDL between patients with cerebral haemorrhage $(0.179(0.023) \mathrm{ng} / \mu \mathrm{g} L D L)$ and the controls. The sex of the subjects did not affect the OxLDL concentration in the patients with cerebral infarction (men, 0.232 (0.021)

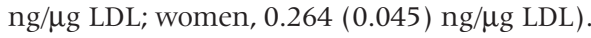

\section{Relations between subtypes of cerebral infarction, NIHSS, and plasma OxLDL}

Next we investigated whether the stroke subtype and the location of the infarct affected the plasma concentration of OxLDL. We found that patients with cardioembolic infarcts (0.273 (0.043) ng/ $\mu \mathrm{g}$ LDL, p < 0.01) and atherothrombotic infarcst $(0.244(0.029) \mathrm{ng} / \mu \mathrm{g}$ LDL, $\mathrm{p}<0.05)$ had significantly higher OxLDL values than the controls. The OxLDL concentrations did not differ between patients with lacunar infarcts (0.182 (0.022) ng/ $\mu \mathrm{g}$ LDL) and the controls. Of the patients with cerebral infarction, those in group GI showed markedly higher OxLDL concentrations $(0.304$ (0.033) ng/ $\mu \mathrm{g}$ LDL) than either the controls or the patients in group GII $(0.169$ (0.012) $\mathrm{ng} / \mu \mathrm{g}$ LDL) $(\mathrm{p}<0.0001$ and $\mathrm{p}<0.001$, respectively) (fig 1 ). Half the GI patients had cardioembolic infarcts while the other half had atherothrombotic infarcts. Figure 2 shows the relation between plasma OxLDL and the NIHSS. Although the plasma OxLDL concentration was not correlated with NIHSS, in GI patients the NIHSS was significantly higher than in GII patients, at $11.6(2.0) \vee 3.5(0.6)(\mathrm{p}<0.01)$, reflecting their markedly increased OxLDL levels.

Effect of age and risk factors on plasma OxLDL

As shown in fig 3, among patients with ischaemic stroke OxLDL was significantly higher than in stratified age matched controls $(p<0.05)$. Furthermore, patients older than 70 years also had higher OxLDL levels than patients younger than 55 years $(p<0.05)$. The incidence of cortical infarction in the cerebral hemisphere tended to increase with age, patients
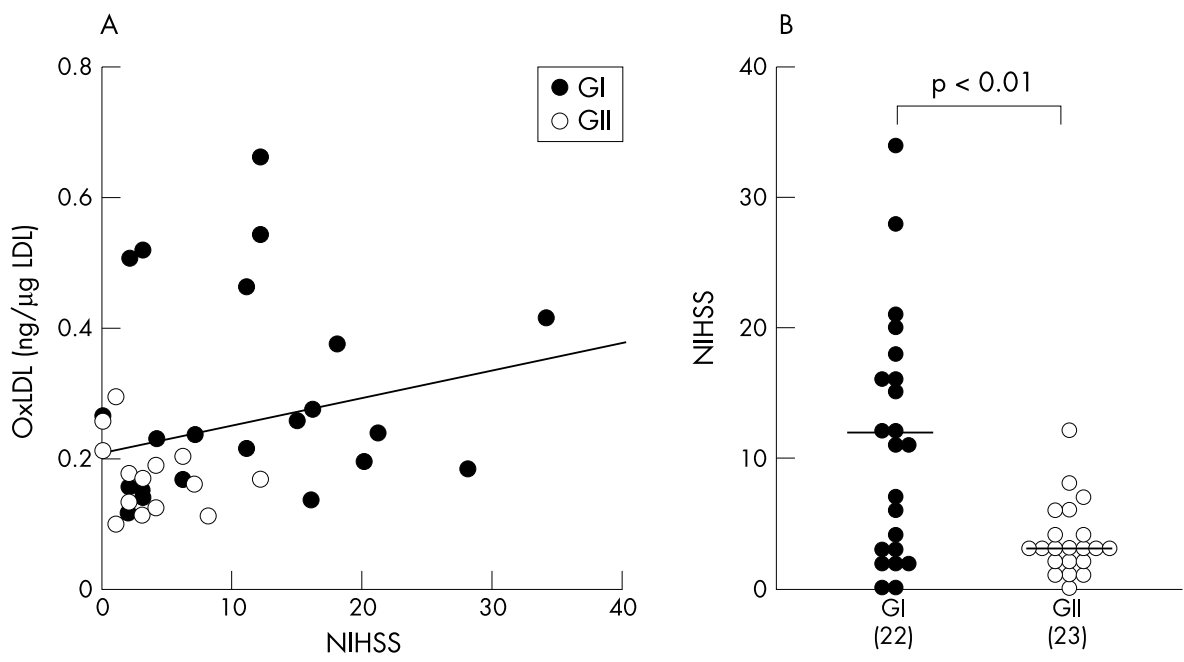

Figure 2 (A) Correlation between plasma oxidised LDL (OxLDL) and the National Institutes of Health stroke scales (NIHSS) in patients with cerebral infarction. (B) NIHSS values in GI infarcts (cortical lesions in cerebral hemisphere) and Gll infarcts (lesions in other regions). Each column and bar indicates the mean and SEM. Statistical analyses were done by the Spearman rank correlation test (A) and the Mann-Whitney $U$ test (B). 

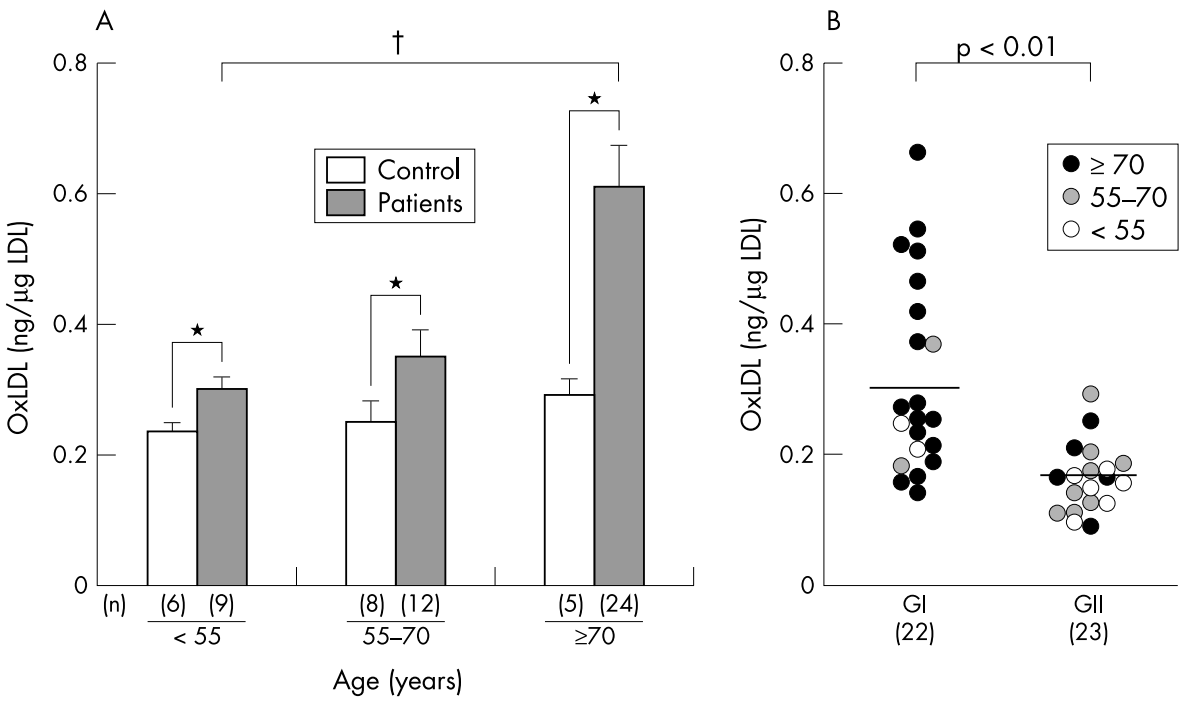

Figure 3 (A) Relation between plasma oxidised LDL (OxLDL) and age in patients with cerebral infarction. Columns and bars indicate the mean and SEM. The Mann-Whitney $U$ test was used for comparisons with the controls ( ${ }^{*} p<0.05$ ), and Scheffé's test for multiple comparisons (†p < 0.05). (B) OxLDL values in Gl infarcts (cortical lesion in cerebral hemisphere) and Gll infarcts (lesion in other regions). OxLDL values between $\mathrm{Gl}$ and $\mathrm{Gll}$ were compared using the $\chi^{2}$ test.

older than 70 years in GI having a markedly high rate $(81.8 \%)$ compared with patients in GII $(26.1 \%)(\mathrm{p}<0.01)$ (fig 3B). This latter finding is likely to be linked to the elevation of plasma OxLDL, suggesting that elderly individuals are at greater risk of severe infarcts than younger persons. On the other hand, we found no significant correlation in our 56 stroke patients between plasma OxLDL and other risk factors such as hypertension, diabetes mellitus, and hyperlipidaemia. As most subjects in this study were smokers, we could not assess the effect of smoking. There were only two patients in our study population who had a history of coronary heart disease; their plasma OxLDL values were was 0.236 and $0.21 \mathrm{l} / \mu \mathrm{g}$ LDL, respectively, and were thus slightly high.

\section{Longitudinal changes of plasma OxLDL after ischaemic stroke}

The changes in OxLDL levels over time during follow up period are shown in fig 4 . Of 45 patients with cerebral infarction, four in group GI died on days $0,3,6$, and 14 days after suffering the insult. Others were transferred to

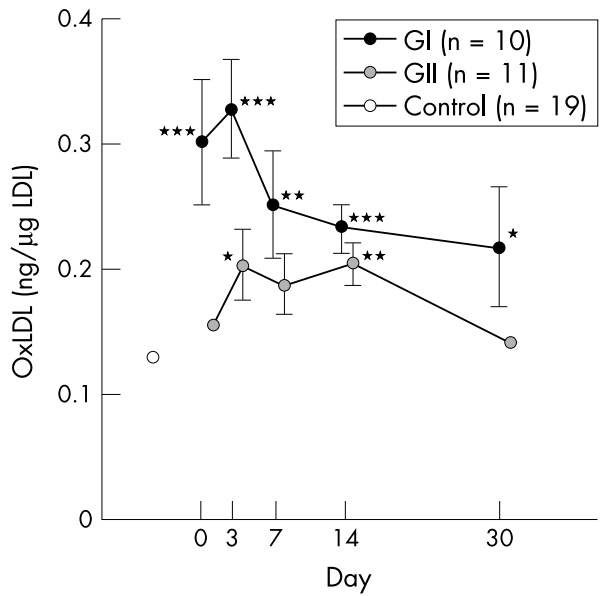

Figure 4 Longitudinal change in the plasma oxidised LDL (OxLDL) level after ischaemic stroke. Gl, cortical lesion in the cerebral hemispheres; GII, infarction in other regions. Data points are means, error bars $=$ SEM. *** $p<0.0001 ; * * p<0.01 ;{ }^{*} p<0.05 v$ control by analysis of variance followed by Scheffé's test. other hospitals and some were discharged or lost to follow up. Thus on the 30th post-event day, 10 GI patients and 11 GII patients were available for determination of plasma OxLDL. Their OxLDL time course is shown in fig 4. In the GI group, plasma OxLDL remained at peak level until the third day after stroke onset. It was markedly higher in the GI patients than in the GII patents, at $0.328(0.039) v 0.197(0.033) \mathrm{ng} / \mathrm{\mu g}$ LDL ( $p<0.01$ ), and it gradually decreased to within the normal range by day 30 (considered a chronic phase). However, in three of $10 \mathrm{GI}$ patients, OxLDL remained elevated even in the chronic phase. While the plasma OxLDL in GII patients was significantly higher than in the controls on day 3 and day 14 after stroke onset, on day 7 and day 30 there was no difference between GII patients and the controls.

\section{DISCUSSION}

In a previous study, Polidori et al reported the involvement of free radicals and lipid peroxidation in human stroke. ${ }^{24}$ However, to our knowledge, this is the first study to demonstrate the association of plasma OxLDL and ischaemic stroke by direct quantitative analysis using monoclonal antibody against oxidised phosphatidylcholine (DLH3).

We showed that patients with cerebral infarction but not those with cerebral haemorrhage had on average almost a twofold increase in OxLDL concentrations compared with controls. In particular, plasma OxLDL was markedly increased in patients with cortical lesions in the cerebral hemisphere (the GI group). This increase peaked on the third day after stroke onset and gradually decreased to within the normal range in the chronic stage. From these findings, it appears that patients with cortical cerebral infarction undergo severe oxidative stress, and plasma OxLDL may be a useful marker for detecting and monitoring the oxidative status of the brain. Of 22 patients with cortical lesions in the cerebral hemisphere, 19 (86\%) had main artery occlusion or stenosis. Our results suggest that the oxidative damage may be extensive. Unfortunately, we did not assess the infarct volume in this study, but our results warrant further studies to explore the relation between plasma OxLDL level and the volume of infarction.

The question arises as to why OxLDL level is raised in cerebral and especially cortical infarction. Ehara et al reported that OxLDL was raised in patients with unstable angina pectoris and acute myocardial infarction who have atherosclerotic lesions. ${ }^{15}$ It is thus possible that a high OxLDL concentration in plasma 
reflects release from the atheromatous plaque. In this study, however, the 22 GI patients were evenly split into those with atherothrombotic and cardioembolic events. Plasma OxLDL in patients with emboli tended to be higher than in those with atherothrombotic events, and patients with embolic strokes usually do not have severe atheromatous plaques in the cerebral main artery. In a previous study, we found that plasma OxLDL concentrations in patients with carotid severe stenosis were significantly higher than in controls, but their plasma OxLDL was not as high as in patients with acute cerebral infarction. ${ }^{25}$ These findings suggest another explanation for the elevation of plasma OxLDL in patients with cerebral infarction: it is reported that lipolysis is increased in brain regions subjected to cerebral ischaemia ${ }^{25}$ and that lipid peroxidation products are increased in the plasma ${ }^{24}$; the source of OxLDL detected by DLH3, recognising oxidised phosphatidylcholine, may in part therefore be oxidised phospholipids released from brain tissue into the circulation.

Another factor to take into account was that OxLDL was significantly higher in stroke patients than in age matched controls, and it continued to increase with age. Patients older than 70 years also had a markedly high incidence of cortical infarcts. It has been reported in previous studies that old people often have dysfunction of their endothelial cells and are vulnerable to oxidative stress. ${ }^{26}{ }^{27}$. LDL oxidation, normally maintained within certain limits by an internal defence system against oxidative stress, may overcome the defences after ischaemic cerebral stroke. As a result, uncontrolled oxidative damage, both in brain cells and in the blood stream, may occur after an insult..$^{28}$ Our data showing that plasma OxLDL continued to increase for three to 14 days after stroke onset in patients with cortical infarction support this hypothesis. In the current study we did not address the function of the antioxidative system or the clearance of OxLDL. We plan to elucidate the mechanism underlying the elevation and recovery of OxLDL in future studies.

We found that in the patients with cortical infarction the rise in plasma OxLDL was correlated with a high NIHSS value. Although plasma OxLDL is not necessarily linked to symptoms, patients with a high level of oxidative stress are at potential risk of extensive brain cell damage after cortical infarcts which may otherwise be silent if the lesions are not in eloquent areas. These findings suggest that plasma OxLDL could be useful as an easily accessible peripheral marker and an aid to prognosis in cerebral infarction, and may supplement information gained by MRI and CT.

There was no significant correlation between plasma OxLDL and risk factors such as hypertension, hyperlipidaemia (even the HDL/LDL ratio), and diabetes mellitus, while other plasma biochemical variables tested did not differ between the patients in the GI and GII groups (data not shown). The possible association between cholesterol levels and stroke risk remains controversial, and a meta-analysis has produced no evidence for such an association. ${ }^{18}$ Until an association is demonstrated, raised plasma OxLDL in patients with ischaemic stroke appears to be a more specific biological marker than conventional risk factors.

\section{Conclusions}

Our study showed a significant association between plasma OxLDL and acute cerebral infarction, especially severe cortical infarction. The findings suggest that a raised OxLDL level reflects oxidative stress in stroke patients and is a useful independent marker of ischaemic stroke.

\section{Authors' affiliations}

M Uno, K T Kitazato, K Nishi, S Nagahiro, Department of

Neurosurgery, School of Medicine, The University of Tokushima, Tokushima, Japan

H Itabe, Department of Microbiology and Molecular Pathology, Faculty of Pharmaceutical Sciences, Teikyo University, Kanagawa, Japan

Competing interests: none declared

\section{REFERENCES}

1 Murray CJ, Lopez AD. Mortality by cause for eight regions of the world: Global Burden of Disease Study. Lancet 1997;349:1269-76.

2 Murray CJ, Lopez AD. Global mortality, disability, and the contribution of risk factors: Global Burden of Disease Study. Lancet 1997:349: 1436-42.

3 Steinberg D. Low density lipoprotein oxidation and its pathobiological significance. J Biol Chem 1997;272:20963-6.

4 Aviram M, Rosenblat M, Etzioni A, et al. Activation of NADPH oxidase required for macrophage-mediated oxidation of low-density lipoprotein. Metabolism 1996;45: 1069-79.

5 Kaplan M, Aviram M. Oxidized low density lipoprotein: atherogenic and proinflammatory characteristics during macrophage foam cell formation. An inhibitory role for nutritional antioxidants and serum paraoxonase. Clin Chem Lab Med 1999:37:777-87.

6 Cherubini A, Mecocci P, Senin U, et al. Autoantibodies against oxidized low-density lipoproteins in older stroke patients. J Am Geriatr Soc 1997:45: 125.

7 McMurray HF, Parthasarathy S, Steinberg D. Oxidatively modified low density lipoprotein is a chemoattractant for human T lymphocytes. J Clin Invest 1993;92: 1004-8.

8 Rajavashisth TB, Andalibi A, Territo MC, et al. Induction of endothelial cell expression of granulocyte and macrophage colony-stimulating factors by modified low-density lipoproteins. Nature 1990;344:254-7.

9 Cushing SD, Berliner JA, Valente AJ, et al. Minimally modified low density lipoprotein induces monocyte chemotactic protein 1 in human endothelial cells and smooth muscle cells. Proc Natl Acad Sci USA 1990:87:5134-8

10 Yla-Herttuala S, Palinski W, Rosenfeld ME, et al. Evidence for the presence of oxidatively modified low density lipoprotein in atherosclerotic lesions of rabbit and man. J Clin Invest 1989:84:1086-95.

11 Itabe $\mathbf{H}$, Takeshima $\mathrm{E}$, Iwasaki $\mathrm{H}$, et al. A monoclonal antibody against oxidized lipoprotein recognizes foam cells in atherosclerotic lesions. Complex formation of oxidized phosphatidylcholines and polypeptides. $J$ Biol Chem 1994;269:15274-9.

12 Itabe H, Yamamoto H, Suzuki $M$, et al. Oxidized phosphatidylcholines that modify proteins. Analysis by monoclonal antibody against oxidized low density lipoprotein. J Biol Chem 1996;271:33208-17.

13 Itabe H, Yamamoto H, Imanaka T, et al. Sensitive detection of oxidatively modified low density lipoprotein using a monoclonal antibody. J Lipid Res 1996;37:45-53.

14 Toshima S, Hasegawa A, Kurabayashi M, et al. Circulating oxidized low density lipoprotein levels: a biochemical risk marker for coronary heart disease. Arterioscler Thromb Vasc Biol 2000;20:2243-7.

15 Ehara S, Ueda M, Naruko T, et al. Elevated levels of oxidized low density lipoprotein show a positive relationship with the severity of acute coronary syndromes. Circulation 2001;103: 1955-60.

16 Holvoet P, Stassen JM, Van Cleemput J, et al. Oxidized low density lipoproteins in patients with transplant-associated coronary artery disease. Arterioscler Thromb Vasc Biol 1998;18:100-7.

17 Holvoet P, Vanhaecke J, Janssens S, et al. Oxidized LDL and malondialdehyde-modified LDL in patients with acute coronary syndromes and stable coronary artery disease. Circulation 1998;98:1487-94.

18 Prospective studies collaboration. Cholesterol, diastolic blood pressure, and stroke: 13,000 strokes in 450,000 people in 45 prospective cohorts. Lancet 1995;46:1647-53.

19 Uno M, Ueda S, Shinno K, et al. Coronary artery stenosis evaluated by combined carotid and coronary angiography in patients undergoing carotid endarterectomy. Neurol Med Chir (Tokyo) 1999:39:567-74.

20 Brott T, Marler JR, Olinger CP, et al. Measurements of acute cerebral infarction: lesion size by computed tomography. Stroke 1989;20:871-5.

21 Goldstein LB, Samsa GP. Reliability of the National Institutes of Health Stroke Scale. Extension to non-neurologists in the context of a clinical trial. Stroke 1997:28:307-10.

22 Adams HP, Bendixen BH, Kappelle $\mathrm{L}$, et al. Classification of subtype of acute ischemic stroke. Definitions for use in a multicenter clinical trial. TOAST: Trial of Org 10172 in Acute Stroke Treatment. Stroke 1993;24:35-41.

23 Bamford J, Sandercock P, Dennis $M$, et al. Classification and natural history of clinical identification subtypes of cerebral infarction. Lancet 1991;337:1521-6.

24 Polidori MC, Frei B, Cherubini A, et al. Increased plasma levels of lipid hydroperoxides in patients with ischemic stroke. Free Radic Biol Med 1998:25:561-7.

25 Nishi K, Itabe H, Uno $M$, et al. Oxidized LDL in carotid plaques and plasma associates with plaque instability. Arterioscl Thromb Vasc Biol 2002;22:1649-54

26 Sun D, Gilboe DD. Ischemia-induced changes in cerebral mitochondrial free fatty acids, phospholipids, and respiration in the rat. J Neurochem 1994;62:1921-8.

27 Rajagopalan S, Brook R, Mehta RH, et al. Effect of losartan in aging-related endothelial impairment. Am J Cardiol 2002;89: 562-6.

28 Taddei S, Virdis A, Ghiadoni L, et al. Age related reduction of NO availability and oxidative stress in humans. Hypertension 2001;38:274-9.

29 Leinonen JS, Ahonen JP, Lonnrot K, et al. Low plasma antioxidant activity is associated with high lesion volume and neurological impairment in stroke. Stroke 2000;31:33-9. 